\title{
GENERATION OF OPTICAL SIGNALS RESISTANT TO DISPERSION-INDUCED POWER DEGRADATION
}

\author{
Vladislav V. Shcherbakov, Anatoly F. Solodkov \\ Center for Fiber-Optic Information Transmission Systems, http://www.centervospi.ru/ \\ 3 Vvedenskogo St., Moscow 117342, Russian Federation
}

Anatoly A. Zadernovsky

MIREA-Russian Technological University (RTU MIREA), http://mirea.ru/

78 Vernadskogo Av., Moscow 119454, Russian Federation

info@centervospi.ru, zadernovsky@mirea.ru

Abstract. A theoretical analysis is performed of transmission of optical signals in a fiber-optic link with external light intensity modulation by an electro-optic dual-drive Mach-Zehnder modulator and direct detection of the signals. The most general analytical expressions are obtained for the optical signals at the output from the modulator and at the receiving end of the fiber-optic link. Special attention is paid to the single-sideband-with-carrier $(\mathrm{SSB}+\mathrm{C})$ and the double-sidebands-withsuppressed-carrier (DSB+SC) modulation formats. A family of new SSB+C modulation formats of dual-drive Mach-Zehnder modulator is determined. Within the framework of linear approximation, the explicit expressions for $\mathrm{SSB}+\mathrm{C}$ and $\mathrm{DSB}+\mathrm{SC}$ optical signals are obtained. Transmission of such signals without dispersion-induced power degradation is clearly demonstrated.

Keywords: fiber-optic links, dual-electrode Mach-Zehnder modulator, fiber chromatic dispersion, single-sideband intensity modulation, suppressed-carrier intensity modulation

PACS: 85.60.Bt

Bibliography -48 references

Received 07.04.2019, accepted 22.04.2019

RENSIT, 2019, 11(2):161-176

DOI: $10.17725 /$ rensit.2019.11.161

\section{Contents}

1. INTRODUCTION (161)

2. Electro-optic Mach-Zehnder MODULATOR (163)

2.1. Electro-Optic EFFECT (163)

2.2. OpERATION PRINCIPLE OF THE ELECTROOPTIC MaCH-ZEHNDER MOdUlator (165)

2.3. BALANCED MACH-ZEHNDER MODULATOR (165)

\subsection{Dual-Drive Mach-Zehnder MODULATOR (167)}

3. Modulation formats of DUAL-Drive MaCH-ZEHNDER MOdUlator (168)

3.1. Double-SIDEBAND-WITH-CARRIER $(\mathrm{DSB}+\mathrm{C})$ MODULATION FORMAT (169)

3.2. SINGLE-SIDEBAND-WITH-CARRIER $(\mathrm{SSB}+\mathrm{C})$ MODULATION FORMAT (169) 3.3. DoubLE-SIDEBAND-WITH-SUPPRESSEDCARRIER (DSB+SC) MODULATION FORMAT (171)

4. Transportation of optical signals OVER A FIBER (171)
4.1. Transportation of the DoubleSIDEBAND-WITH-CARRIER (DSB+C) MODULATION SIGNAL (172)

4.2. Transportation of the SingleSIDEBAND-WITH-CARRIER (SSB+C) MODULATION SIGNAL (172)

4.3. Transportation of THE DOUbleSIDEBAND-WITH-SUPPRESSED-CARRIER (DSB+SC) MODULATION SIGNAL (173)

5. Conclusion (173)

REFERENCES (174)

\section{INTRODUCTION}

Analog fiber-optic transmission systems remain in demand for various applications. Among them: on-board fiber-optic networks, antenna systems remote from the control and signal processing points, signal distribution systems for either on-board radars or ground-based radars with phased antenna arrays, radioover-fiber systems, CATV networks, remote 
cellular antenna service systems and many others. Standard fiber-optic link includes a transmitter, which converts an input electrical signal into a signal of intensity modulation of light produced by a semiconductor laser, transportation of the optical signal over a fiber and, finally, direct detection of the signal by a photodiode at the receiving end of the fiber. There are two basic schemes for the light intensity modulation, which compete with regard to simplicity and performance. The first scheme uses direct intensity modulation, which can be produced by varying the drive current of the semiconductor laser. Unfortunately, this current variation not only modulates the laser light intensity, but induces a parasitic optical frequency modulation, the effect which is referred to as frequency chirp.

The second scheme was proposed as a solution to the problem of chirp. This scheme employs external intensity modulation of the laser light by a balanced electro-optic MachZehnder modulator (MZM), which produces the chirp-free optical signals. Unfortunately, the fiber-optic link with balanced MZM suffers from severe degradation of light intensity modulation response. At certain modulation frequencies, transmission of the optical signals is completely suppressed. Such behavior is typical, for the fiber-optic link with externally modulated 1550 $\mathrm{nm}$ laser light source and standard single-mode optical fiber. It is caused by chromatic dispersion of the group velocity of electromagnetic waves in the fiber.

As an optical signal is transported over a fiber, the different spectral components of modulated electromagnetic wave are traveling, due to dispersion, with different velocities. As a consequence, they form at the fiber output a superposition of waves with the phase relationships which differ from that of in the input signal. This leads to a distortion of the transmitted signal. At first approximation, the detected signal of light intensity at the modulation frequency can be regarded as a result of interference of two waves of beats between the first order and the minus first order sidebands and the carrier electromagnetic wave. At the receiving end of the fiber these beating waves acquire, because of dispersion, different phase shifts, the magnitude of which depends on the modulation frequency. In particular, at certain modulation frequencies, these waves turn out to be in opposite phases, so that destructive interference occurs and the output signal is completely suppressed. Such a modulation format is referred to as the double-sidebandwith-carrier $(\mathrm{DSB}+\mathrm{C})$ modulation.

To overcome the dispersion-induced power degradation, a special modulation format is used to generate optical single-sideband signals containing only one of the two spectral sidebands near to the carrier. It is referred to as the singlesideband-with-carrier $(\mathrm{SSB}+\mathrm{C})$ modulation format. In the absence of second sideband, the single wave of beats simply does not have a pair for the interference.

Another possibility is to use the doublesidebands-with-suppressed-carrier (DSB+SC) modulation format. In this case, there are no two waves of beats between the sidebands and the carrier, due to absence of the latter. Accordingly, the signal of light intensity results from the beats between the upper and the lower sidebands. The beat frequency is twice the modulation frequency. Since only single wave of beats occurs, DSB+SC optical signal is not affected by the dispersioninduced power degradation.

Various techniques for generating such optical signals have been repeatedly discussed in the literature. For instance, to eliminate one of the two spectral sidebands and, thus, to obtain $\mathrm{SSB}+\mathrm{C}$ modulation signal, the frequency filtering was employed in [1-3]. In some schemes, special operation modes of a dual-drive electro-optic Mach-Zehnder modulator [4, 5] or an electroabsorption modulator [6-8] were employed. In others, direct modulation of a injection-locked semiconductor laser was used [9-12]. 
The carrier wave suppression techniques for generation of DSB+SC signals of double modulation frequency were considered in [1317], and further development of these techniques with the aim of obtaining the 4-tupled frequency signals was proposed in [18-21], the 6-tupled frequency signals in [22], the 8-tupled signals in [23], the 12-tupled signals in [24-26] and the 16-tupled signals in [27].

Evolution of the methods for generating $\mathrm{SSB}+\mathrm{C}$ optical signals has led to development of the single-sideband-with-suppressed-carrier $(\mathrm{SSB}+\mathrm{SC})$ modulation format [28-31]. The main advantages of this format are in improved spectral and energy efficiency of signal transmission and in tolerance to dispersive signal distortions. The disadvantages include significant complication of the processes of signal generation and detection. In order to demodulate SSB+SC signal, an additional copy of the carrier wave is necessary to have at the receiving end of the fiber-optic link, since the original carrier, according to the format name, is not transmitted over the fiber. The photodetector registers the signal of beats between the local carrier and the sideband received over the fiber.

The most effective and simplest way of generating the optical signals free of dispersion-induced power degradation is the use of dual-drive electro-optic Mach-Zehnder modulator [4, 5]. Study of the operation modes of such a modulator in a fiber-optic link was carried out by many authors. In some works [32], the calculations are limited to small-signal approximation, which involves into consideration only two harmonics at the fundamental frequency. A more general analysis, which takes into account an entire spectral composition, was performed in [33]. However, the results of this analysis are presented in the form of infinite series. This drawback was overcome in [34], where summation of the series was performed and more compact expressions were obtained (a generalization to a modulator with asymmetric arms is presented in [35]). Nevertheless, the obtained formulas are still cumbersome and hard to engineering perception. Matching of these formulas with more simple expressions, obtained in the small signal approximation, is difficult, since the calculations were performed separately for even and odd spectral components.

In this paper, we will obtain the most general analytical expressions for optical signals at the output from the modulator and at the receiving end of a fiber-optic link. Despite the entire spectral composition of the signals is taken into account, the final formulas are quite compact and make it possible straightforward matching with the approximate expressions for a small modulation depth. Based on the performed calculations, a family of new $\mathrm{SSB}+\mathrm{C}$ modulation formats will be determined. Within the framework of linear approximation, the explicit expressions for $\mathrm{SSB}+\mathrm{C}$ and $\mathrm{DSB}+\mathrm{SC}$ optical signals will be obtained and the transmission of such signals without dispersion-induced power degradation will be clearly demonstrated.

\section{ELECTRO-OPTIC MACH-ZEHNDER MODULATOR}

\subsection{ELECTRO-OPTIC EFFECT}

Electro-optic modulators are widely used in modern optical communication systems. The physics behind the operation of such modulators is the electro-optic Pockels effect. In a broad sense, the electro-optic effect [36] consists in changing the optical properties of some materials in response to applying a constant or slowly varying (compared to the frequency of the light) electric field. In particular, certain materials change their refractive index when subjected to an electric field. For classification of the electrooptic effect, we expand the dependence of refractive index $n$ on electric field strength $\hat{E}$ in a power series

$$
n=n_{0}+a_{1} \hat{E}+\left(a_{2} / 2\right) \hat{E}^{2}+\ldots
$$


where $a_{n}=\left(d^{n} n / d \hat{E}^{n}\right)_{\hat{E}=0}$. If the third term can be neglected, then $n$ is a linear function of the electric field strength $\hat{E}$ (Pockels effect). The linear electro-optic effect can occur only in the crystals with non-centrosymmetric lattice. Among them: potassium dihydrogen phosphate $\mathrm{KH}_{2} \mathrm{PO}_{4}(\mathrm{KDP})$, barium titanate $\mathrm{BaTiO}_{3}$, lithium niobate $\mathrm{LiNbO}_{3}$, lithium tantalate $\mathrm{LiTaO}_{3}$ and $\mathrm{A}^{\mathrm{III}} \mathrm{B}^{\mathrm{V}}$ semiconductors, such as gallium arsenide - GaAs, indium phosphide - InP and others. Lithium niobate, gallium arsenide and indium phosphide are the most often materials used for manufacturing of Mach-Zehnder modulators. If the coefficient $a_{1}=0$, the quadratic dependence of the refractive index $n$ on the electric field strength $\hat{E}^{2}$ is advanced to the forefront (Kerr effect).

In crystal optics, it is assumed to consider, instead of (1), a series

$$
\frac{1}{n^{2}}=\frac{1}{n_{0}^{2}}+r \hat{E}+\xi \hat{E}^{2}+\ldots,
$$

where electro-optic coefficients $r=-2 a_{1} / n_{0}^{3}$ and $\xi=-a_{2} / n_{0}^{3}$ are known as Pockels and Kerr coefficients, respectively. Typical values of Pockels coefficients $r$ are in the range of $10^{-12} \div 10^{-10} \mathrm{~m} / \mathrm{V}$, and typical values of the Kerr coefficients $\xi$ are $10^{-18} \div 10^{-14} \mathrm{~m}^{2} / \mathrm{V}^{2}$ in solid-state materials and $10^{-22} \div 10^{-19} \mathrm{~m}^{2} / \mathrm{V}^{2}$ in liquids.

Lithium niobate is the most popular and widely used material exhibiting linear electrooptic effect [37]. It is optically transparent in the wavelength range of 400-5000 $\mathrm{nm}$. Lithium niobate is an uniaxial crystal, this crystallographic axis is usually denoted by the letter Z. Lithium niobate is anisotropic crystal its Pockels coefficient depends on the direction of the external electric field strength and on the direction of propagation and polarization of the light wave. The Pockels effect in lithium niobate is most pronounced when the external electric field is directed along the $Z$-axis of the crystal (the Pockels coefficient is $30 \mathrm{pm} / \mathrm{V}$ ) and the electromagnetic wave propagates along the crystallographic $Y$-axis with polarization in the $Z Y$ plane. For the manufacture of MachZehnder modulators, monocrystalline lithium niobate plates, cut perpendicularly to one of the crystallographic axes are used. The most widespread are two types of modulators: with $Z$-cut plates and $X$-cut plates.

Organic polymers occupy a special position among electro-optic materials [38]; they have a number of advantages over inorganic crystals. Polymers differ in chemical diversity, relative simplicity of synthesis, ease of processing, and high values of electro-optic coefficients. If in inorganic crystals, the Pockels coefficient varies in the range of $1 \div 100 \mathrm{pm} / \mathrm{V}$, then for organic polymers it reaches the values of $100 \div 500$ pm/V.

The electro-optical properties of polymers are due to chromophores, which are special organic molecules that have an electric dipole moment. Chromophores can be introduced into polymer matrix either as impurities or as a part of the side or main chain of the polymer material. In order for the polymer becomes noncentrosymmetric and, thus, is capable to exhibit the electro-optic Pockels effect, it is subjected to so-called poling. During this process, the polymer is heated to a high temperature, at which local mobility of the chromophores and segments of the polymer chains appears. Then the polymer is placed in a strong electric field. In this field, the electric dipole moments of the chromophores become oriented in the direction of the vector of electric field strength. Further, the polymer is cooled without turning off the electric field, and, thereby, a material with "frozen" macroscopic polarization is obtained. For electro-optic devices, polymeric materials are used with a long-term orientation conservation of the chromophore dipoles in the polymer matrix.

The electro-optic effect is practically noninertial. In inorganic crystals, the response time for Pockels effect consists $10^{-10} \div 10^{-11} \mathrm{~s}$, and in polymer materials $10^{-12} \div 10^{-13} \mathrm{~s}$. Thus, 
the upper limit for the modulation frequency of the refractive index can reach hundreds of gigahertz for inorganic crystals, and for electrooptic polymers it falls into a terahertz frequency range.

Electro-optic modulators according to the scheme of the Mach-Zehnder interferometer [39-41] are the most widely used in fiberoptic transmission systems. The operation of such modulators is based on the transverse Pockels effect (the external electric field is perpendicular to the direction of light propagation), and lithium niobate is usually used as a material.

\subsection{Operation principle of the electro- OPTIC MACH-ZEHNDER MOdULATOR}

A schematic diagram of Mach-Zehnder modulator is shown in Fig. 1. The modulator includes a system of optical waveguide channels with single input and single output, which are formed on a monocrystalline lithium niobate substrate by thermal diffusion of titanium. The input optical channel splits into two independent waveguides, providing a spatial separation of the input laser radiation into two light beams, which, then, are combined together in the output channel. The drive electrodes are mounted on the substrate. A voltage, applied to the electrodes, creates an electric field, which penetrates into the waveguides cross sections. This field changes the optical path length of the waveguides. The drive voltages $U_{1}$ and $U_{2}$ govern the phase shifts $\varphi_{1}$ and $\varphi_{2}$, acquired each of the light waves on passing through their own waveguides.

The input electromagnetic wave with the frequency $\omega_{0}$ and the amplitude $E_{0}$ is split in the modulator with symmetric waveguide geometry

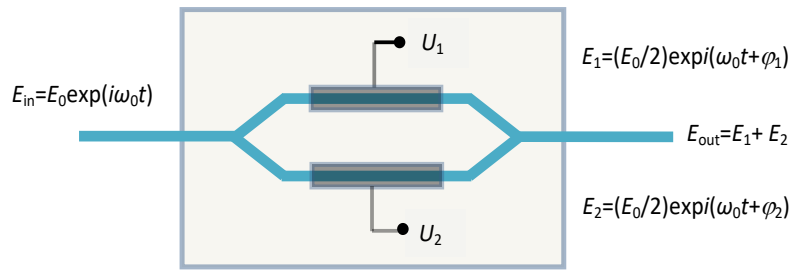

Fig. 1. Schematic diagram of electro-optic Mach-Zehnder modulator. into two waves of equal amplitude $E_{0} / 2$. At the modulator output we obtain

$$
\begin{aligned}
& E_{\text {out }}=\left(E_{0} / 2\right) \exp i\left(\omega_{0} t+\varphi_{1}\right)+ \\
& +\left(E_{0} / 2\right) \exp i\left(\omega_{0} t+\varphi_{2}\right)= \\
& =E_{0} \exp \left(i \omega_{0} t\right) \cos \left[\left(\varphi_{1}-\varphi_{2}\right) / 2\right] \exp \left[i\left(\varphi_{1}+\varphi_{2}\right) / 2\right]
\end{aligned}
$$

Since the output light intensity $P_{\text {out }}$ is proportional to $\left|E_{\text {out }}\right|^{2}$ then

$$
P_{\text {out }}=P_{0} \cos ^{2}\left[\left(\varphi_{1}-\varphi_{2}\right) / 2\right] \text {. }
$$

Varying the drive voltages $U_{1}$ and $U_{2}$ leads to a modulation of the light intensity at the output of the modulator, and the corresponding electromagnetic wave (3) proves to be modulated, in general, not only in amplitude, but also in phase. Thus, the optical signal at the output of the modulator suffers from a frequency chirp. In a particular case, when $\varphi_{1}=-\varphi_{2}$, the phase modulation of the light electromagnetic wave disappears and the modulator operates in a mode of amplitude modulation without chirp. If $\varphi_{1}=$ $\varphi_{2}$, then there is no amplitude modulation, and the modulator operates in a mode of phase modulation.

The mode of chirp-free amplitude modulation can be achieved with antiphase variation of the controlling electric fields strength in the channel waveguides. This can be ensured by a special arrangement of the electrodes with an employment of single drive electrode common to both waveguides. Such a modulator is called as a single-drive or balanced Mach-Zehnder modulator.

\subsection{Balanced Mach-Zehnder modulator}

A schematic diagram of the balanced MZM is shown in Fig. 2, and its cross-section in Fig. 3. Such a modulator automatically provides antiphase changes in the strength vectors of controlling electric fields in channel waveguides. Due to linearity of Pockels effect, the signs of the refractive index changes in the channel waveguides will be opposite to each other. In order for the controlling electric field strength to be directed along the $Z$-axis (direction of the strongest Pockels effect), 


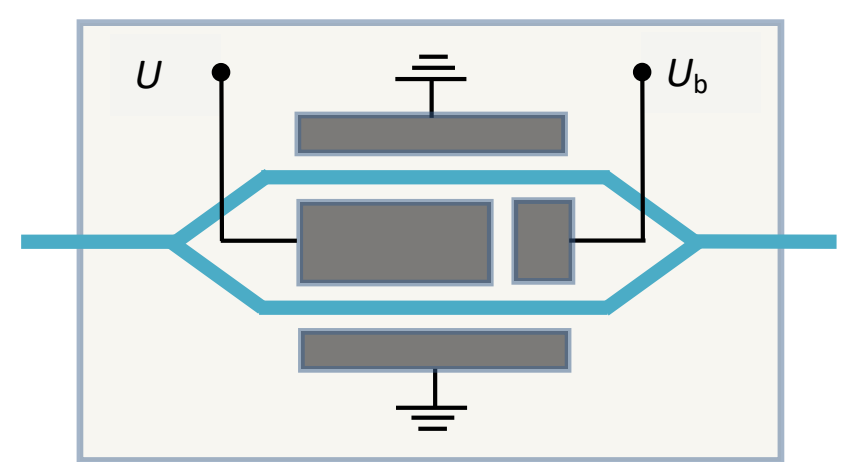

Fig. 2. Schematic diagram of the balanced Mach-Zebnder modulator.

the $X$-cut lithium niobate crystals are used as a substrate.

With the help of (1) the relation between the drive voltage $U$ and the phases $\varphi_{1}$ and $\varphi_{2}$ can be written as

$$
\begin{gathered}
\varphi_{1}=\frac{2 \pi l}{\lambda_{0}} n_{0}+\frac{2 \pi l}{\lambda_{0}} a_{1} \alpha U, \\
\varphi_{2}=\frac{2 \pi l}{\lambda_{0}} n_{0}-\frac{2 \pi l}{\lambda_{0}} a_{1} \alpha U,
\end{gathered}
$$

where $\lambda_{0}$ is the wavelength of light, $l$ is the length of channel waveguides of the modulator. It is assumed that the strengths of the controlling electric fields in the cross sections of the waveguides are proportional to the applied voltage $U$, and the coefficient of proportionality $\alpha$ is the same for each channel and depends only on the geometry of the electrodes location. Such an assumption is justified only when the modulator electrodes are arranged symmetrically relative to the channel waveguides. The minus sign in the relation (6) is due to the fact that the electric fields strength vectors in the waveguides are directed oppositely (see Fig. 3). Due to linearity of the Pockels effect, the reversal in

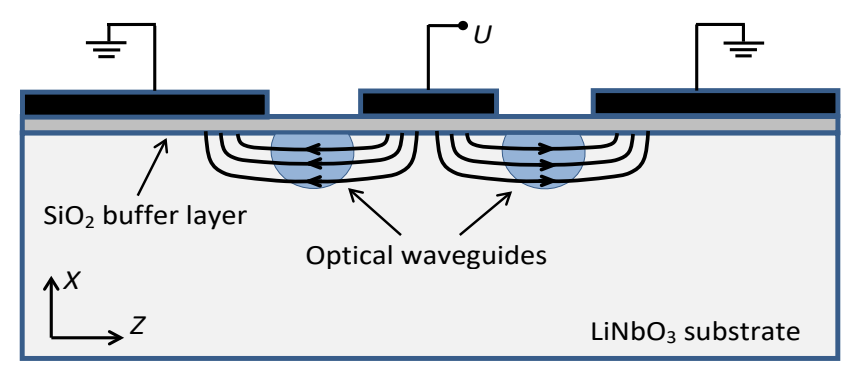

Fig. 3. Cross-section of the balanced Mach-Zehnder modulator. direction of the applied electric field, results in the reversal in sign of the refractive index change.

For the quantitative characteristics of the Pockels effect, the voltage $U=U_{\pi}$ is used, at which the phase difference between (5) and (6) will be equal to $\pi$. With such a voltage, the electromagnetic waves at the interferometer output cancel each other and the light intensity becomes equal to zero. The voltage $U_{\pi}$ is specific to each device and it is called the half-wave voltage of the modulator or the switch-off voltage. With this parameter, the electromagnetic wave (3) takes the form

$$
E=E_{0} \exp i\left(\omega_{0} t+\varphi_{0}\right) \cos \frac{\pi}{2} \frac{U}{U_{\pi}},
$$

and the intensity of light (4) at the output of the modulator can be written as (we neglect the insertion loss)

$$
P_{\text {out }}=P_{0} \cos ^{2} \frac{\pi}{2} \frac{U}{U_{\pi}} \text {. }
$$

The plot of the relative intensity $P_{\text {out }} / P_{0}$ versus the normalized voltage $U / U_{\pi}$ is shown in Fig. 4 . It is called the transfer function of modulator. It can be seen from the figure that small voltage modulations near the operating points with $U$ $= \pm U_{\pi} / 2$ should lead to a linear modulation of the light intensity. The choice of the operating point of modulator is achieved with the help of a constant bias voltage, which introduces an additional controlled phase shift between the

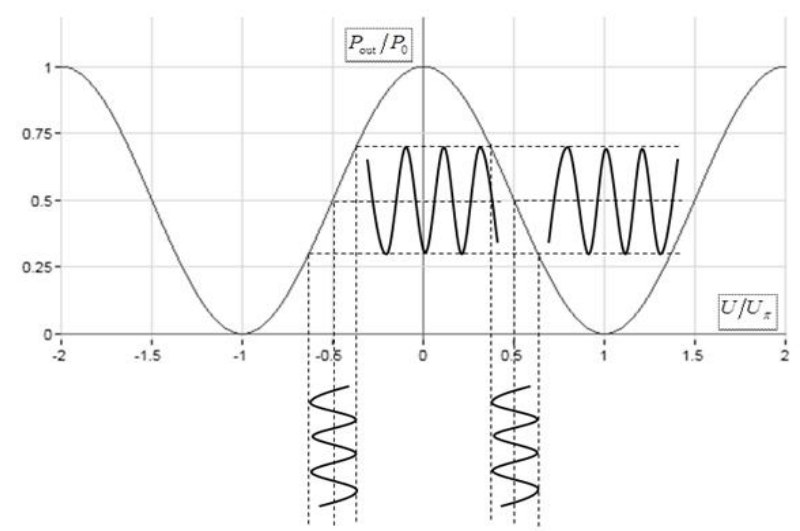

Fig. 4. Plot of the relative light intensity $P_{\text {out }} / P_{0}$ at the output of modulator versus the normalized drive voltage $U / U_{\pi}$. Transfer function of modulator. 
light waves in the arms of the interferometer. To ensure biasing, a separate set of electrodes is usually used (see Fig. 2).

One of the main requirements to operation of the Mach-Zehnder modulator is the minimum of introduced nonlinear distortions. It is necessary for use in transmission systems with high sensitivity and large dynamic range. In general, the conversion of an electrical signal into an optical one with the help of MachZehnder modulator is non-linear. Indeed, let the total drive voltage $U$ (taking into account the bias voltage) be harmonically modulated

$$
U / U_{\pi}=u_{\mathrm{b}}+u_{0} \cos \left(\omega_{\mathrm{m}} t+\varphi_{\mathrm{m}}\right)
$$

with normalized bias voltage $u_{\mathrm{b}}$, normalized modulation amplitude $u_{0}$, modulation frequency $\omega_{\mathrm{m}}$ and initial phase $\varphi_{\mathrm{m}}$. Then, the electromagnetic wave (7) at the output of the modulator can be represented as a spectral sum

$$
E=E_{0} e^{i\left(\omega_{0} t+\varphi_{0}\right)} \sum_{n=-\infty}^{+\infty} C_{n} e^{i n\left(\omega_{\mathrm{m}} t+\varphi_{\mathrm{m}}\right)}
$$

with the coefficients

$$
C_{n}=\cos \left(\frac{\pi u_{\mathrm{b}}}{2}+n \frac{\pi}{2}\right) J_{n}\left(\pi u_{0} / 2\right)
$$

The spectral representation of the light intensity modulation signal (8) at the output of the modulator,

$$
\begin{aligned}
& P_{\text {out }}= \\
& =\left(P_{0} / 2\right)\left[1+\sum_{n=-\infty}^{+\infty} \cos \left(\pi u_{\mathrm{b}}+n \frac{\pi}{2}\right) J_{n}\left(\pi u_{0}\right) e^{i n\left(\omega_{\mathrm{m}} t+\varphi_{\mathrm{m}}\right)}\right]
\end{aligned}
$$

reveals the presence not only the first order harmonics at the fundamental frequency $\omega_{m}$ but also all the higher order harmonics. Here $J_{n}$ is the Bessel function of the first kind of order $n$.

The degree of non-linearity can be reduced by selecting the operating point of modulator. Indeed, at the normalized bias voltages $u_{\mathrm{b}}=$ $m+1 / 2$, where $m=0, \pm 1, \pm 2, \pm 3, \ldots$, (so called quadrature operating points) all the even harmonics in (12) disappear and only the fundamental harmonics at the modulation frequency $\omega_{\mathrm{m}}$ and all the higher odd harmonics of this frequency are present in the output optical signal. The power of these harmonics at the normalized voltage amplitude, for example, $u_{0}=1 / 2$ (this corresponds to $100 \%$ depth of modulation at the normalized bias voltage $u_{0}=$ $\pm 1 / 2)$ are proportional to $J_{1}(\pi / 2)=0.567, J_{3}(\pi / 2)$ $=6.894 \times 10^{-2}$ and $J_{5}(\pi / 2)=2.240 \times 10^{-3}$, so that the power of the third harmonic amounts to $-9.15 \mathrm{~dB}$ of the fundamental harmonic power, whereas the power of the fifth harmonic is $-24.03 \mathrm{~dB}$. At a smaller signal modulation with $u_{0}=1 / 4$ we obtain $J_{1}(\pi / 4)=0.363, J_{3}(\pi / 4)=$ $9.696 \times 10^{-3}, J_{5}(\pi / 4)=7.566 \times 10^{-5}$, so that the third and the fifth harmonic powers are only $-15.73 \mathrm{~dB}$ and $-36.8 \mathrm{~dB}$, accordingly. One can see, therefore, that at $\pi u_{0}<1$ we can restrict ourselves only by the fundamental harmonics. In this approximation, the power of the output optical signal is reduced to

$$
P_{\text {out }}=P_{0}\left[1 / 2+(-1)^{m+1} J_{1}\left(\pi u_{0}\right) \cos \left(\omega_{\mathrm{m}} t+\varphi_{\mathrm{m}}\right)\right],
$$

and it is worth noting that at even $\mathrm{m}$ the signal of light intensity modulation is in opposite phases with the drive voltage signal (9), whereas at odd $\mathrm{m}$ these signals are in-phase. Taking for definiteness that $u_{\mathrm{b}}= \pm 1 / 2$, which gives the minimum magnitude of the bias voltage, we obtain

$$
P_{\text {out }}=\left(P_{0} / 2\right)\left[1 \mp \pi u_{0} \cos \left(\omega_{\mathrm{m}} t+\varphi_{\mathrm{m}}\right)\right] \text {, }
$$

where it is taken into account that in the region $\pi u_{0}<1$, one can accept $J_{1}\left(\pi u_{0}\right) \approx \pi u_{0} / 2$. Thus, the quadrature biased balanced Mach-Zehnder modulator provides a chirp-free amplitude modulation of the carrier wave with the minimal non-linear distortions of the light intensity modulation signal.

\subsection{Dual-drive MaCh-Zehnder modulator}

The Mach-Zehnder modulator can be designed to have two separate signal electrodes and to control the electric fields in the arms of the interferometer independently of each other. A schematic diagram of such a modulator is shown in Fig. 5, and its cross-section - in Fig. 6. It is called a dual-drive MZM. Here, to achieve 


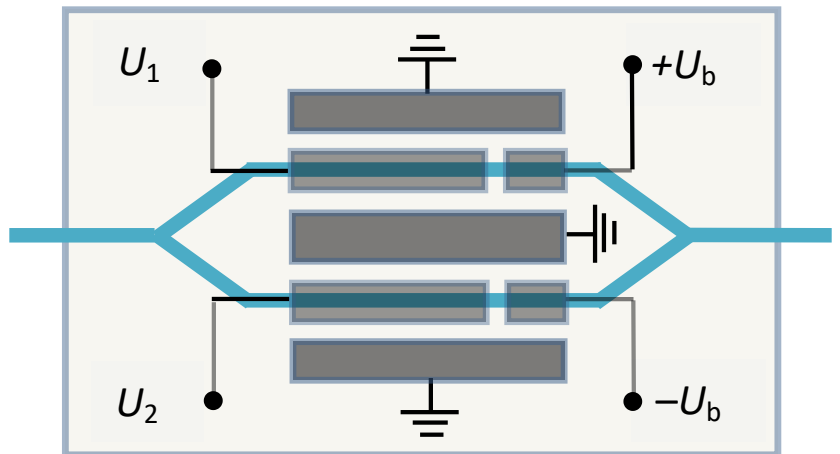

Fig. 5. Schematic diagram of the dual-drive Mach-Zehnder modulator.

maximum electro-optic effect, the $Z$-cut lithium niobate crystals are used as a substrate.

It is worth noting that a buffer dielectric layer is coated between the electrodes and the substrate surface, as shown in Fig. 3, 6. In the case of $Z$-cut modulator, the presence of this layer is obligatory, since the electromagnetic field of an optical wave, goes, as a rule, beyond the channel waveguide and in the absence of the dielectric layer between the crystal surface and the metal, a strong attenuation of the optical radiation appears due to large losses in metal electrodes. In the case of $X$-cut modulator, the buffer layer is used to better matching between the speed of the traveling microwaves of driving voltage and the speed of light in the channel waveguides. Such speed matching plays a key role in increasing the frequency modulation bandwidth.

Despite the high modulation rate of the refractive index (about of $100 \mathrm{GHz}$ ) inherent in lithium niobate, the operating frequency range of the Mach-Zehnder modulators is much smaller because of a number of physical limitations. To understand these limitations, we

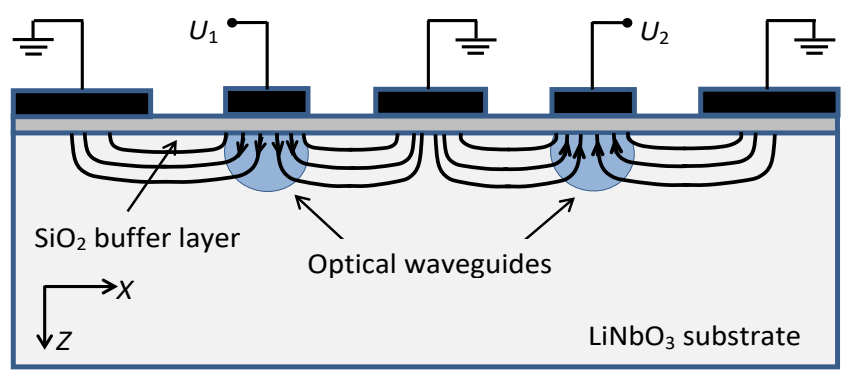

Fig. 6. Cross-section of the dual-drive Mach-Zehnder modulator. note that the typical magnitudes of changes in the refractive index caused by the driving electric field are much smaller than the refractive index itself. Therefore, to achieve effective modulation, one has to either increase the driving voltage or increase the interaction length of the electrical and optical fields in the channel waveguides. Large electric voltages are undesirable because they lead to an increase in non-linear distortion of the optical signal. An increase in the interaction length is achieved by using the traveling-wave electrodes, in which the electric signal propagates in the same direction as the optical wave. In this case, it is necessary that the speeds of light and the traveling electric wave coincide. It is the mismatch between the phase velocities of microwaves and optical waves that determines the practical limit of the modulation bandwidth. Reducing the mismatch is achieved by decreasing the effective dielectric constant with the help of the buffer dielectric layer between the electrodes and the substrate, which leads to an increase in the phase velocity of the microwaves. In addition, the design optimization of the modulator electrodes, aimed at reducing the attenuation of microwaves, also contributes to an increase in the effective interaction length. As a result, in modern modulators based on lithium niobate crystals, the $-3 \mathrm{~dB}$ modulation bandwidth amounts to $30-50 \mathrm{GHz}$. In the polymer-based modulators with inherent faster Pockels effect the bandwidth reaches $100 \mathrm{GHz}$ and more.

Dual-drive Mach-Zehnder modulator provides a wide variety of modulation formats. In particular, it can operate in a mode of the balanced modulator, if the signal electrodes are supplied with the same, but antiphase voltage.

\section{MODULATION FORMATS OF DUAL-DRIVE MACH-ZEHNDER MODULATOR}

We consider in more detail the operation of a dual-drive Mach-Zehnder modulator. Let us apply to signal electrodes the driving 
voltages $U_{1}=U_{0} \cos \left(\omega_{\mathrm{m}} t+\varphi_{\mathrm{m} 1}\right)$ and $U_{2}=$ $U_{0} \cos \left(\omega_{\mathrm{m}} t+\varphi_{\mathrm{m} 2}\right)$ with the equal frequencies $\omega_{\mathrm{m}}$, the equal amplitudes $U_{0}$, but with different initial phases $\varphi_{\mathrm{m} 1}$ and $\varphi_{\mathrm{m} 2}$. The operating point can be selected by applying to the bias electrodes an equal constant voltage $U_{b}$ with opposite polarity (see Fig. 5). Such a voltage produces the equal in magnitude, but opposite in sign, phase shifts of light waves in the arms of the interferometer. When $U_{b}=U_{\pi}$ the modulator does not pass the light, since the corresponding electromagnetic waves are in antiphase and cancel each other at the output from the modulator. As a result, the phases of electromagnetic waves in the arms of the interferometer can be written as

$$
\begin{aligned}
& \varphi_{1}=\varphi_{0}+\pi U_{b} / 2 U_{\pi}+\pi U_{1} / 2 U_{\pi} \\
& \varphi_{2}=\varphi_{0}-\pi U_{b} / 2 U_{\pi}+\pi U_{2} / 2 U_{\pi}
\end{aligned}
$$

and the electromagnetic light wave at the exit of the modulator is

$$
E=E_{0} \exp i\left(\omega_{0} t+\varphi_{0}\right) \cos \frac{\pi}{2}\left(\frac{U_{\mathrm{b}}}{U_{\pi}}+\frac{U_{1}-U_{2}}{2 U_{\pi}}\right) \exp \frac{i \pi}{4} \frac{U_{1}+U_{2}}{U_{\pi}} .
$$

The spectral representation of the wave (17) can be written as a sum

$$
E=E_{0} \mathrm{e}^{i\left(\omega_{0} t+\varphi_{0}\right)} \sum_{n=-\infty}^{+\infty} C_{n} e^{i n\left(\omega_{\mathrm{m}} t+\frac{\varphi_{\mathrm{m} 1}+\varphi_{\mathrm{m} 2}}{2}\right)}
$$

with the coefficients

$$
C_{n}=i^{n} J_{n}\left(\pi u_{0} / 2\right) \cos \left(\frac{\pi u_{\mathrm{b}}}{2}+n \frac{\varphi_{\mathrm{m} 1}-\varphi_{\mathrm{m} 2}}{2}\right),
$$

where normalized variables $u_{\mathrm{b}}=U_{\mathrm{b}} / U_{\pi}$ and $u_{0}$ $=U_{0} / U_{\pi}$ are introduced again. Accordingly, the spectral representation of the signal of light intensity modulation at the output of the modulator is

$$
\begin{aligned}
& P_{\text {out }}=\frac{P_{0}}{2}\left[1+\sum_{n=-\infty}^{+\infty} i^{n} J_{n}\left(\pi u_{0} \sin \frac{\varphi_{\mathrm{m} 1}-\varphi_{\mathrm{m} 2}}{2}\right) \times\right. \\
& \left.\times \cos \left(\pi u_{\mathrm{b}}+n \frac{\pi}{2}\right) e^{i n\left(\omega_{\mathrm{m}} t+\frac{\varphi_{\mathrm{m} 1}+\varphi_{\mathrm{m} 2}}{2}\right)}\right] .
\end{aligned}
$$

With an antiphase variation of the signal voltages, when $\varphi_{\mathrm{m} 1}-\varphi_{\mathrm{m} 2}= \pm \pi$, the dual-drive modulator switches to an operation mode of the balanced modulator, and formulas $(18) \div(20)$, as it should be, turn into $(10) \div(12)$.

We consider the modulation formats that are most commonly used in analog transmission systems.

\subsection{DOUBLE-SIDEBAND-WITH-CARRIER}

\section{$(\mathrm{DSB}+\mathrm{C})$ MODULATION FORMAT}

The format parameters: $\varphi_{\mathrm{m} 1}=\varphi_{\mathrm{m}},\left|\varphi_{\mathrm{m} 1}-\varphi_{\mathrm{m} 2}\right|$ $=\pi, u_{\mathrm{b}}= \pm 1 / 2, \pi u_{0}<1$. Dual-drive modulator operates in a mode of the quadrature biased balanced modulator. The signal of intensity modulation (20) contains only odd harmonics. At small modulation depths, the optical signal is described by the function

$$
P=\left(P_{0} / 2\right)\left[1 \mp \pi u_{0} \cos \left(\omega_{\mathrm{m}} t+\varphi_{\mathrm{m}}\right)\right],
$$

and the corresponding electromagnetic wave (17) does not suffer from the frequency chirp, it has a spectral composition (19) and contains three main spectral components: the carrier wave with the amplitude $C_{0}=1 / \sqrt{2}$ and two sidebands with the amplitudes $C_{1}=C_{-1}=i\left(\pi u_{0} / 4\right) C_{0}$ at $\varphi_{\mathrm{m} 1}$ $-\varphi_{\mathrm{m} 2}=+\pi, u_{\mathrm{b}}=-1 / 2$, or at $\varphi_{\mathrm{m} 1}-\varphi_{\mathrm{m} 2}=-\pi$, $u_{\mathrm{b}}=+1 / 2$; and with the amplitudes $C_{1}=C_{-1}=$ $-i\left(\pi u_{0} / 4\right) C_{0}$ at $\varphi_{\mathrm{m} 1}-\varphi_{\mathrm{m} 2}=+\pi, u_{\mathrm{b}}=+1 / 2$, or at $\varphi_{\mathrm{m} 1}-\varphi_{\mathrm{m} 2}=-\pi, u_{\mathrm{b}}=-1 / 2$. As it should be, the expression (21) is obviously matched to (14).

\subsection{SingLE-SIDEBAND-WITH-CARRIER (SSB+C) MODULATION FORMAT}

From (19) it is easy to see that the condition that the harmonic with, for example, $n=-1$ disappears from the light wave spectrum looks like

$$
u_{\mathrm{b}}-\frac{\varphi_{\mathrm{m} 1}-\varphi_{\mathrm{m} 2}}{\pi}=(2 p+1), \quad p=0, \pm 1, \pm 2, \ldots
$$

Taking into account that the range of the bias voltage $-1 \leq u_{\mathrm{b}} \leq 1$ exhausts all kinds of operating points of modulator, and the interval $-1 \leq\left(\varphi_{\mathrm{m} 1}-\varphi_{\mathrm{m} 2}\right) / \pi \leq 1$ gives all possible phase differences of driving voltages, we obtain, instead of (22), the condition 


$$
\left|u_{\mathrm{b}}-\frac{\varphi_{\mathrm{m} 1}-\varphi_{\mathrm{m} 2}}{\pi}\right|=1 .
$$

Similarly, the condition that the harmonic with $n=+1$ disappears is determined by the equality

$\left|u_{\mathrm{b}}+\frac{\varphi_{\mathrm{m} 1}-\varphi_{\mathrm{m} 2}}{\pi}\right|=1$.

It should be noted that at $u_{\mathrm{b}}=0$ and fulfillment of the above conditions (23) or (24), all the odd harmonics are completely disappeared together with the harmonics -1 or +1 . At $u_{\mathrm{b}}=$ \pm 1 and fulfillment of the condition (23) or (24), modulation of light intensity does not occur. These extreme cases should be excluded from the consideration.

Table 1 presents examples of the singlesideband modulation formats obtained with the help of the requirements (23), (24). Some of them are well known. For instance, the format $u_{\mathrm{b}}= \pm 1 / 2,\left(\varphi_{\mathrm{m} 1}-\varphi_{\mathrm{m} 2}\right) / \pi= \pm 1 / 2$ was historically the first proposed single-sideband optical modulation format $[4,5]$. Another format with $u_{\mathrm{b}}= \pm 1 / 3,\left(\varphi_{\mathrm{m} 1}-\varphi_{\mathrm{m} 2}\right) / \pi= \pm 2 / 3$ was proposed relatively recently [42].

Not all formats included in the family (23), (24) are equivalent. From (20), for example, one can see that the choice of the quadrature operating points $u_{\mathrm{b}}= \pm 1 / 2$, located on linear segment of the modulator transfer function,

Table

Examples of the single-sideband modulation formats

\begin{tabular}{|c|c|c|}
\hline $\begin{array}{c}\text { Harmonic } \\
\text { number with } \\
\text { zero amplitude } \\
n\end{array}$ & $\begin{array}{c}\text { Normalized bias } \\
\text { voltage } \\
u_{\mathrm{b}}=U_{\mathrm{b}} / U_{\pi}\end{array}$ & $\begin{array}{c}\text { Normalized phase } \\
\text { difference of drive voltages } \\
\left(\varphi_{\mathrm{m} 1}-\varphi_{\mathrm{m} 2}\right) / \pi\end{array}$ \\
\hline \multirow{4}{*}{-1} & $-1 / 2$ & $+1 / 2$ \\
\cline { 2 - 3 } & $+1 / 2$ & $-1 / 2$ \\
\cline { 2 - 3 } & $-1 / 3$ & $+2 / 3$ \\
\cline { 2 - 3 } & $+1 / 3$ & $-2 / 3$ \\
\cline { 2 - 3 } & $-2 / 3$ & $+1 / 3$ \\
\cline { 2 - 3 } & $+2 / 3$ & $-1 / 3$ \\
\hline \multirow{5}{*}{+1} & $-1 / 2$ & $-1 / 2$ \\
\cline { 2 - 3 } & $+1 / 2$ & $+1 / 2$ \\
\cline { 2 - 3 } & $-1 / 3$ & $-2 / 3$ \\
\cline { 2 - 3 } & $+1 / 3$ & $+2 / 3$ \\
\cline { 2 - 3 } & $-2 / 3$ & $-1 / 3$ \\
\cline { 2 - 3 } & $+2 / 3$ & $+1 / 3$ \\
\hline
\end{tabular}

lead to suppression of all even harmonics of the intensity modulation signal. Thus, in this case the nonlinear distortions of the signals at the output of the modulator are minimal. Another interesting example [42] is the format $u_{\mathrm{b}}=-1 / 3$, $\left(\varphi_{\mathrm{m} 1}-\varphi_{\mathrm{m} 2}\right) / \pi=+2 / 3$, or $u_{\mathrm{b}}=+1 / 3,\left(\varphi_{\mathrm{m} 1}-\right.$ $\left.\varphi_{\mathrm{m} 2}\right) / \pi=-2 / 3$ for which not only the harmonic $n=-1$ disappears but also the harmonic $n=$ +2 . And if one selects the following modulation parameters $u_{\mathrm{b}}=-1 / 3,\left(\varphi_{\mathrm{m} 1}-\varphi_{\mathrm{m} 2}\right) / \pi=-2 / 3$, or $u_{\mathrm{b}}=+1 / 3,\left(\varphi_{\mathrm{m} 1}-\varphi_{\mathrm{m} 2}\right) / \pi=+2 / 3$ the harmonics $n=+1$ and $n=-2$. disappear simultaneously. It is seen, that in this case the modulation becomes "single-sideband" with regard to not only the fundamental, but also to the second harmonic. As a result, we should expect a decrease in nonlinear distortions of the signal transmission over dispersive fiber.

As an illustration, we consider a few examples in more detail. Let the modulation format $\varphi_{\mathrm{m} 1}$ $=\varphi_{\mathrm{m}}, \varphi_{\mathrm{m} 2}=\varphi_{\mathrm{m}} \pm \pi / 2, u_{\mathrm{b}}= \pm 1 / 2, \pi u_{0}<1$ is employed. Two cases can be calculated separately.

1) If $\varphi_{\mathrm{m} 1}=\varphi_{\mathrm{m}}, \varphi_{\mathrm{m} 2}=\varphi_{\mathrm{m}}+\pi / 2, u_{\mathrm{b}}= \pm 1 / 2$, then the optical intensity modulation signal is described in linear approximation by the function $P_{\text {out }}=\frac{P_{0}}{2}\left[1 \mp \frac{\pi u_{0}}{\sqrt{2}} \cos \left(\omega_{\mathrm{m}} t+\varphi_{\mathrm{m}}-\frac{\pi}{4}\right)\right]$,

and the corresponding electromagnetic wave has a spectral composition (19), which contains the following main spectral components: the carrier wave with the amplitude $C_{0}=1 / \sqrt{2}$ and the sidebands with the amplitudes $C_{1}=0, C_{-1}=$ $-i\left(\pi u_{0} / 4\right) C_{0}$ at $u_{\mathrm{b}}=-1 / 2$ or $C_{1}=i\left(\pi u_{0} / 4\right) C_{0}, C_{-1}$ $=0$ at $u_{\mathrm{b}}=+1 / 2$.

2) If $\varphi_{\mathrm{m} 1}=\varphi_{\mathrm{m}}, \varphi_{\mathrm{m} 2}=\varphi_{\mathrm{m}}-\pi / 2, u_{\mathrm{b}}= \pm 1 / 2$, then the optical intensity modulation signal is given by

$$
P_{\text {out }}=\frac{P_{0}}{2}\left[1 \mp \frac{\pi u_{0}}{\sqrt{2}} \cos \left(\omega_{\mathrm{m}} t+\varphi_{\mathrm{m}}+\frac{\pi}{4}\right)\right],
$$

and the electromagnetic wave has a spectral composition (19) with the following main spectral components: the carrier wave with the amplitude $C_{0}=1 / \sqrt{2}$ and the sidebands with 
the amplitudes $C_{1}=i\left(\pi u_{0} / 4\right) C_{0}, C_{-1}=0$ at $u_{\mathrm{b}}=$ $-1 / 2$; or $C_{1}=0, C_{-1}=-i\left(\pi u_{0} / 4\right) C_{0}$, at $u_{\mathrm{b}}=+1 / 2$.

Now, we choose the modulation format $\varphi_{\mathrm{m} 1}$ $=\varphi_{\mathrm{m}}, \varphi_{\mathrm{m} 2}=\varphi_{\mathrm{m}} \pm 2 \pi / 3, u_{\mathrm{b}}= \pm 1 / 3, \pi u_{0}<1$. Two cases can be considered.

1) If $\varphi_{\mathrm{m} 1}=\varphi_{\mathrm{m}}, \varphi_{\mathrm{m} 2}=\varphi_{\mathrm{m}}+2 \pi / 3, u_{\mathrm{b}}= \pm 1 / 3$, then the optical intensity modulation signal takes the form

$$
P_{\text {out }}=\frac{3 P_{0}}{4}\left[1 \mp \frac{\pi u_{0}}{\sqrt{2}} \cos \left(\omega_{\mathrm{m}} t+\varphi_{\mathrm{m}}-\frac{\pi}{6}\right)\right],
$$

2) If $\varphi_{\mathrm{m} 1}=\varphi_{\mathrm{m}}, \varphi_{\mathrm{m} 2}=\varphi_{\mathrm{m}}-2 \pi / 3, u_{\mathrm{b}}= \pm 1 / 3$, then the optical intensity modulation signal looks like

$$
P_{\text {out }}=\frac{3 P_{0}}{4}\left[1 \mp \frac{\pi u_{0}}{\sqrt{2}} \cos \left(\omega_{\mathrm{m}} t+\varphi_{\mathrm{m}}+\frac{\pi}{6}\right)\right],
$$

\subsection{Double-SIDEBAND-WITH-SUPPRESSED- CARRIER (DSB+SC) MODULATION FORMAT}

If the minima of the modulator transfer function are used as operating points, for example the points with $u_{\mathrm{b}}= \pm 1$, then, according to (19), the carrier amplitude $C_{0}$ is equal to zero. At a variety of phases of the driving voltages, we obtain a family of modulation formats with suppressed carrier. The optical intensity modulation signal (20) takes the form

$$
\begin{aligned}
& P_{\text {out }}=\frac{P_{0}}{2}\left[1-\sum_{n=-\infty}^{+\infty} i^{n} J_{n}\left(\pi u_{0} \sin \frac{\varphi_{\mathrm{m} 1}-\varphi_{\mathrm{m} 2}}{2}\right) \times\right. \\
& \left.\times \cos \left(n \frac{\pi}{2}\right) e^{i n\left(\omega_{\mathrm{m}} t \varphi_{\varphi_{\mathrm{m}}+\varphi_{\mathrm{m} 2}}\right)}\right]
\end{aligned}
$$

which contains only even harmonics. In particular, at a small modulation depth, $\pi u_{0}<1$, we obtain

$$
\begin{aligned}
& P_{\text {out }}=\frac{P_{0}}{2}\left(\frac{\pi u_{0}}{2}\right)^{2} \sin ^{2} \frac{\varphi_{\mathrm{m} 1}-\varphi_{\mathrm{m} 2}}{2} \times \\
& \times\left[1-\cos 2\left(\omega_{\mathrm{m}} t+\frac{\varphi_{\mathrm{m} 1}+\varphi_{\mathrm{m} 2}}{2}\right)\right],
\end{aligned}
$$

where the following approximate relations $J_{0}(\mathrm{z})$ $\approx 1-(\mathrm{z} / 2)^{2}, J_{2}(\mathrm{z}) \approx(1 / 2)(\mathrm{z} / 2)^{2}$ were used. It is seen that at the modulator output an optical signal with double modulation frequency is generated.

As an illustration, we consider the modulation format $\varphi_{\mathrm{m} 1}=\varphi_{\mathrm{m}}, \varphi_{\mathrm{m} 2}=\varphi_{\mathrm{m}} \pm \pi, u_{\mathrm{b}}= \pm 1$. In this case, the dual-drive modulator operates in a mode of the balanced modulator and the spectrum of the corresponding electromagnetic wave (18) contains only odd harmonics. The main components of this spectrum are: $C_{0}=0$, $C_{1}=C_{-1}= \pm i\left(\pi u_{0} / 4\right)$ at $\varphi_{\mathrm{m} 1}=\varphi_{\mathrm{m}}, \varphi_{\mathrm{m} 2}=\varphi_{\mathrm{m}}+$ $\pi, u_{\mathrm{b}}= \pm 1$; or $C_{0}=0, C_{1}=C_{-1}=\mp i\left(\pi u_{0} / 4\right)$ at $\varphi_{\mathrm{m} 1}=\varphi_{\mathrm{m}}, \varphi_{\mathrm{m} 2}=\varphi_{\mathrm{m}}-\pi, u_{\mathrm{b}}= \pm 1$. Provided that $\pi u_{0}<1$, we obtain the following light intensity modulation signal

$$
P_{\text {out }}=\frac{P_{0}}{2}\left(\frac{\pi u_{0}}{2}\right)^{2}\left[1+\cos 2\left(\omega_{\mathrm{m}} t+\varphi_{\mathrm{m}}\right)\right] \text {. }
$$

\section{TRANSPORTATION OF OPTICAL SIGNALS OVER A FIBER}

Due to chromatic dispersion, each spectral wave from the composition (18) travels in a fiber at its own velocity. This leads to a distortion of the optical signal at the output of the fiber. Indeed, after passing the distance $L$, a spectral wave with a frequency $\omega$ takes the form $E=E_{0} \mathrm{e}^{i(\omega t \beta L L)}$, where $\beta$ is the propagation constant at the specified frequency (we neglect the attenuation). Dispersion is expressed in dependence of the propagation constant on frequency. In the vicinity of the carrier frequency $\omega_{0}$ the function $\beta(\omega)$ can be represented as a power series $\beta(\omega)=\beta_{0}+\beta_{1}\left(\omega-\omega_{0}\right)+\left(\beta_{2} / 2\right)(\omega-$ $\left.\omega_{0}\right)^{2}+\ldots$, where $\beta_{0}$ is the propagation constant at the carrier frequency, $\beta_{1}=1 / u$ is equal to the inverse group velocity, $u=d \omega / d \beta$, at the carrier frequency and the coefficient $\beta_{2}$ is associated with the group velocity dispersion. It can be determined through the so-called chromatic dispersion coefficient $D$ of a fiber by means of the well-known relation [43] $\beta_{2}=-\lambda_{0}^{2} D / 2 \pi c$, where $c$ - the speed of light in vacuum, $\lambda_{0}-$ laser emission wavelength. Typical values for $D$ at the wavelength $1550 \mathrm{~nm}$ are in the range (16 -18) ps/(nm km). 
As a result, at the output of a fiber of length $L$, each spectral component in (18) acquires its own phase shift equal to $-\beta L$. In particular, for the harmonic with the frequency $\omega_{0}+n \omega_{\mathrm{m}}$ it consists $\beta L=-\beta_{0} L-\beta_{1} n \omega_{\mathrm{m}} L-(1 / 2) \beta_{2} n^{2} \omega_{\mathrm{m}}{ }^{2} L-\ldots$. Therefore, at the fiber output we have the wave

$$
E=E_{0} e^{i\left(\omega_{0} t-\beta_{0} L+\varphi_{0}\right)} \sum_{n=-\infty}^{+\infty} C_{n} e^{i n^{2} \theta} e^{i\left[\omega_{\mathrm{m}}(t-L / u)+\frac{\varphi_{\mathrm{m} 1}+\varphi_{\mathrm{m} 2}}{2}\right]},
$$

where

$$
\theta=\pi c\left(\omega_{\mathrm{m}} / \omega_{0}\right)^{2} D L
$$

is the signal transportation parameter.

The intensity of light at the output from the optical fiber (and, consequently, the photocurrent of detector) will be proportional to the square modulus of the electric field strength $E$ (32). We write down it in the form

$$
P_{\mathrm{s}}=\sum_{n=-\infty}^{+\infty} P_{n} e^{i\left[\omega_{\mathrm{m}}(t-L / u)+\frac{\varphi_{\mathrm{m} 1}+\varphi_{\mathrm{m} 2}}{2}\right]},
$$

where

$$
\begin{aligned}
& P_{n}=P_{0} \sum_{k=-\infty}^{+\infty} C_{n+k} e^{i(n+k)^{2} \theta} C_{k}^{*} e^{-i k^{2} \theta}= \\
& =P_{0} \sum_{k=-\infty}^{+\infty} C_{n+k} e^{i(n+k) n \theta} C_{k}^{*} e^{i k n \theta}
\end{aligned}
$$

is the complex amplitude of the harmonic with frequency $n \omega_{\mathrm{m}}$. In calculating (35), we use the Graf's theorem on summation of the Bessel functions (Graf's addition theorem) [44], which, for the purpose of our analysis, can be written in the form [45]

$$
J_{n}\left(z_{3}\right) e^{i n \gamma}=\sum_{k=-\infty}^{+\infty} J_{n+k}\left(z_{1}\right) e^{i(n+k) a} J_{k}\left(z_{2}\right) e^{i k \beta},
$$

where the complex number $z_{3} \mathrm{e}^{i \eta}$ is the sum of two other complex numbers $z_{1} e^{i a}$ and $-z_{2} e^{-i \beta}$. As a result, we obtain the expression

$$
\begin{aligned}
& P_{n}=(-1)^{n} \frac{P_{0}}{2}\left\{J_{n}\left(\pi u_{0} \sin n \theta\right) \cos \frac{n\left(\varphi_{m 1}-\varphi_{m 2}\right)}{2}+\right. \\
& +\frac{1}{2} e^{i \pi u_{b}} J_{n}\left[\pi u_{0} \sin \left(n \theta+\frac{\left(\varphi_{m 1}-\varphi_{m 2}\right)}{2}\right)\right]+ \\
& \left.+\frac{1}{2} e^{-i \pi u_{b}} J_{n}\left[\pi u_{0} \sin \left(n \theta-\frac{\left(\varphi_{m 1}-\varphi_{m 2}\right)}{2}\right)\right]\right\} .
\end{aligned}
$$

Simplified version of (37) which is suitable to the balanced Mach-Zehnder modulator was obtained earlier in [46].

\subsection{Transportation OF THE DOUBLE-SIDEBAND- WITH-CARRIER (DSB+C) MODULATION SIGNAL}

After transportation over a dispersive fiber of length $L$, the signal (21) is transformed to the form

$$
P_{\mathrm{s}}=\left(P_{0} / 2\right)\left[1 \mp \pi u_{0} \cos \theta \cos \left(\omega_{\mathrm{m}}(t-L / u)+\varphi_{\mathrm{m}}\right)\right] .
$$

When $\cos \theta$ goes to zero, the signal at the fiber output disappears $[47,48]$. This happens at the modulation frequencies

$$
\omega_{\mathrm{m}}=\omega_{0} \sqrt{\frac{k+1 / 2}{c D L}}, k=0,1,2, \ldots
$$

A typical value of the fiber dispersion coefficient $D=17 \mathrm{ps} /(\mathrm{nm} \cdot \mathrm{km})$, the laser light wavelength $1550 \mathrm{~nm}$ corresponds to the frequency $f_{0}=\omega_{0} / 2 \pi=193.414$ ТГц.

Thus, the use of this modulation format leads to significant performance degradation of the fiber-optic link. At modulation frequencies (39), transmission of the optical signals is completely suppressed.

\subsection{TRANSPORTATION OF THE SINGLE-SIDEBAND-} WITH-CARRIER (SSB+C) MODULATION SIGNAL

At the output from fiber of length $L$, the signals (25), (26) and (27), (28) are transformed, respectively, to the forms

$$
\begin{aligned}
& P_{\mathrm{s}}=\frac{P_{0}}{2}\left\{1 \mp \frac{\pi u_{0}}{\sqrt{2}} \cos \left[\omega_{\mathrm{m}}(t-L / u)+\varphi_{\mathrm{m}}-\frac{\pi}{4} \pm \theta\right]\right\}, \\
& P_{\mathrm{s}}=\frac{P_{0}}{2}\left\{1 \mp \frac{\pi u_{0}}{\sqrt{2}} \cos \left[\omega_{\mathrm{m}}(t-L / u)+\varphi_{\mathrm{m}}+\frac{\pi}{4} \mp \theta\right]\right\},
\end{aligned}
$$

and

$$
\begin{aligned}
& P_{\mathrm{s}}=\frac{3 P_{0}}{4}\left\{1 \mp \frac{\pi u_{0}}{2} \cos \left[\omega_{\mathrm{m}}(t-L / u)+\varphi_{\mathrm{m}}-\frac{\pi}{6} \pm \theta\right]\right\}, \\
& P_{\mathrm{s}}=\frac{3 P_{0}}{4}\left\{1 \mp \frac{\pi u_{0}}{2} \cos \left[\omega_{\mathrm{m}}(t-L / u)+\varphi_{\mathrm{m}}+\frac{\pi}{6} \mp \theta\right]\right\} .
\end{aligned}
$$

A comparison of these expressions shows that the $\mathrm{SSB}+\mathrm{C}$ modulation signal transmission does not suffer from the amplitude distortions. The corresponding signals at the input and at the output of a fiber differ only in phase. 
4.3. Transportation of THE DoubleSIDEBAND-WITH-SUPPRESSED-CARRIER

(DSB+SC) MODULATION SIGNAL

At the receiving end of a fiber-optic link the signal (30) takes the form

$$
\begin{aligned}
& P_{\mathrm{s}}=\frac{P_{0}}{2}\left(\frac{\pi u_{0}}{2}\right) \sin ^{2} \frac{\left(\varphi_{\mathrm{m} 1}-\varphi_{\mathrm{m} 2}\right)}{2} \times \\
& \times\left\{1-\cos 2\left[\omega_{\mathrm{m}}(t-L / u)+\frac{\left(\varphi_{\mathrm{m} 1}-\varphi_{\mathrm{m} 2}\right)}{2}\right]\right\},
\end{aligned}
$$

and its particular version (31), corresponding to a mode of balanced modulator, is transformed to

$$
P_{\mathrm{s}}=\frac{P_{0}}{2}\left(\frac{\pi u_{0}}{2}\right)^{2}\left\{1+\cos 2\left[\omega_{\mathrm{m}}(t-L / u)+\varphi_{\mathrm{m}}\right]\right\} .
$$

A comparison of signals at the input and at the output of a fiber indicates that the signals under consideration are transmitted without amplitude distortions. Moreover, the output signal (44) is completely independent of the parameter of transportation $\theta$ (33).

\section{CONCLUSION}

When a light intensity modulation signal travels through a fiber, its power is periodically degraded (this phenomenon is also referred to as signal power fading or signal power penalty). The spatial period of such degradation depends on frequency of the signal. As a result, signals of certain frequencies cannot be transmitted over a fiber of a given length. The reason for such a behavior lies in the chromatic dispersion of the group velocity of electromagnetic waves in the fiber.

In this paper, the special signals of light intensity modulation resistant to dispersioninduced power degradation are considered. Among them, the single-sideband-with-carrier and the double-sideband-with-suppressedcarrier modulation signals. For both kind of signals, only a single electromagnetic wave of beats is formed for which there is no a pair wave for destructive interference. The easiest way to generate such signals is to use a dual-drive MachZehnder modulator. The most general analytical expressions for the optical signals at the output from the modulator and at the receiving end of a fiber-optic link were obtained. These expressions allow us to determine the contribution of any higher order harmonics and can be used to analyze various signal distortions. Based on the performed calculations, a family of new singlesideband modulation formats of the dual-drive Mach-Zehnder modulator was determined

In the framework of linear approximation, the explicit expressions were obtained for the single-sideband-with-carrier and the doublesideband-with-suppressed-carrier modulation signals. Transmission of such signals without dispersion-induced power degradation was clearly demonstrated. In other words, it was shown that the $\mathrm{SSB}+\mathrm{C}$ and $\mathrm{DSB}+\mathrm{SC}$ modulation signals are resistant to amplitude distortions. The spectral amplitudes of these signals at the input and at the output of a fiber remain equal in magnitude (without taking into account the attenuation in the fiber). As for phase distortions, these signals behave differently.

The signals of $\mathrm{SSB}+\mathrm{C}$ modulation at the input (25) - (28) and at the output (40) - (43) of a fiber differ in phase by an amount, which contains the parameter $\theta$. Since the signal transportation parameter $\theta$ (33) is a quadratic function of the modulation frequency, the phase-frequency characteristic of the fiber-optic link is non-linear. This leads to violation of the phase relationships between spectral components of the transmitted signal and, thus, to the signal distortion.

The output DSB+SC modulation signal (44) is not dependent on the signal transportation parameter $\theta$. Therefore, such a signal is resistant not only to the amplitude, but also to the phase distortions. It is necessary, however, to bear in mind that under employment of such modulation format, the conversion of an electrical signal into an optical one occurs with frequency doubling.

\section{ACKNOWLEDGEMENTS}

This work was partly supported by the Ministry of Science and Education of the Russian Federation, Project No. 8.4853.2017/BCh. 


\section{REFERENCES}

1. Park J, Sorin WV, Lau KY. Elimination of the fibre chromatic dispersion penalty on 1550 $\mathrm{nm}$ millimetre-wave optical transmission. Electron. Lett., 1997, 33:512-513.

2. Yonenaga K, Takachio N. A fiber chromatic dispersion compensation technique with an optical SSB transmission in optical homodyne detection systems. IEEE Photon. Technol. Lett., 1993, 5:949-951.

3. Vourch E, Le Berre D, Herve D. Lightwave single sideband wavelength self-tunable filter using InP:Fe crystal for fiber-wireless systems. IEEE Photon. Technol. Lett., 2002, 14:194-196.

4. Smith GH, Novak D, Ahmed Z. Technique for optical SSB generation to overcome dispersion penalties in fibre-radio systems. Electron. Lett., 1997, 33(1):74-75.

5. Smith G, Novak D, Ahmed Z. Overcoming chromatic-dispersion effects in fiber-wireless systems incorporating external modulators. IEEE Transactions on microwave theory and techniques, 1997, 45(8):1410-1415.

6. Vergnol E, Devaud F, Tanguy D, Penard E. Integrated Lightwave Millimetric Single Sideband Source: Design and Issues. IEEE Journal of Lightwave Technology, 1998, 16(7):1276-1284.

7. Polo V, Marti J, Ramos F, Moodie D. Mitigation of chromatic dispersion effects employing electroabsorption modulatorbased transmitters. IEEE Photon. Technol. Lett., 1999, 7:883-885.

8. Fells JAJ, Gibbon MA, White IH, Thompson GHB, Penty RV, Armistead CJ, Kimber EM, Moule DJ, Thrush EJ. Transmission beyond the dispersion limit using a negative chirp electroabsorption modulator. Electron. Lett., 1994, 30:1168-1169.

9. Hyuk-Kee Sung, Erwin KLau, Ming CWu. Optical single sideband modulation using strongopticalinjection-locked semiconductor lasers. IEEE Photonics Technology Letters, 2007, 19(13):1005-1007.
10. Arahira S, Matsui Y, Kunii T, Oshiba S, Ogawa Y. Transform-limited optical shortpulse generation at high repetition rate over $40 \mathrm{GHz}$ from a monolithic passive modelocked DBR laser diode. IEEE Photon. Technol. Lett., 1993, 5:1362-1365.

11. Nirmalathas A, Liu HF, Ahmed Z, Novak D, Ogawa Y. Subharmonic synchronous and hybrid mode-locking of a monolithic DBR laser operating at millimeter-wave frequencies. IEEE Photon. Technol. Lett., 1997, 9(4):434-436.

12. Kim DY, Pelusi M, Ahmed Z, Novak D, Liu HF, Ogawa Y. Ultrastable millimetre-wave signal generation using hybrid modelocking of a monolithic DBR laser. Electron. Lett., 1995, 31(9):733-734.

13. O’Reilly JJ, Lane PM, Heidemann R, Hofstetter R. Optical generation of very narrow linewidth millimetre wave signals. Electron. Lett., 1992, 28(25):2309-2311.

14. Montgomery R, De Salvo R. A novel technique for double sideband suppressed carrier modulation of optical fields. IEEE Photonics Technology Letters, 1995, 7(4):434-436.

15. Griffin RA, Lane PM, O’Reilly JJ. Dispersiontolerant subcarrier data modulation of optical millimetre-wave signals. Electron. Lett., 1996, 32:2258-2260.

16. Fuster JM, Marti J, Corral JL. Chromatic dispersion effects in electro-optical upconverted millimetre-wave fibre optic links. Electron. Lett., 1997, 33:1969-1970.

17. Jia Z, Yu J, Chang G-K. A full-duplex radioover-fiber system based on optical carrier suppression and reuse. IEEE Photon. Technol. Lett., 2006, 18:1726-1728.

18. O'Reilly JJ, Lane PM. Fibre-supported optical generation and delivery of $60 \mathrm{GHz}$ signals. Electron. Lett., 1994, 30(16):1329-1330.

19. Yu J, Jia Z, Yi L, Wang T, Chang GK. Centralized lightwave radio-over-fiber system with photonic frequency quadrupling for high frequency millimeter-wave generation. IEEE Photon. Technol. Lett., 2007, 19(19):1499-1501. 
20. Lin CT, Shih PT, Chen J, Xue WQ, Peng PC, Chi S. Optical millimeter-wave signal generation using frequency quadrupling technique and no optical filtering. IEEE Photon. Technol. Lett., 2008, 20(12):1027-1029.

21. Mohamed M, Zhang X, Hraimel B, Wu K. Analysis of frequency quadrupling using a single Mach-Zehnder modulator for millimeter-wave generation and distribution over fiber systems. Optics Express, 2008, 16(14):10786-10802.

22. Qin Y, Sun J. Frequency sextupling technique using two cascaded dual-electrode MachZehnder modulators interleaved with Gaussian optical band-pass filter. Optics Communications, 2012, 285(12):2911-2916.

23. Zhang H, Cai L, Xie S, Zhang K, Wu X, Dong Z. A novel radio-over-fiber system based on carrier suppressed frequency eightfold millimeter wave generation. IEEE Photonics Journal, 2017, 9(5):1-6.

24. Shih PT, Chen J, Lin CT, Jiang WJ, Huang HS, Peng PC, Chi S. Optical millimeter-wave signal generation via frequency 12-Tupling. IEEE Journal of Lightwave Technology, 2010, 28(1):71-78.

25. Zhu Z, Zhao S, Li Y, Chu X, Wang X, Zhao $\mathrm{G}$. A radio-over-fiber system with frequency 12-tupling optical millimeter-wave generation to overcome chromatic dispersion. IEEE Journal of Quantum Electronics, 2013, 49(11):919-922.

26. Dongfei Wang, Xianfeng Tang, Yichen Fan, Xiaoguang Zhang, Lixia Xi, Wenbo Zhang. A new approach to generate the optical millimeter-wave signals using frequency 12-tupling without an optical filter. Proc. SPIE Tenth International Conference on Information Optics and Photonics, 2018, $109641 \mathrm{C}$.

27. Dongfei Wang, Xianfeng Tang, Yichen Fan, Xiaoguang Zhang, Lixia Xi, Wenbo Zhang. A novel filterless scheme for 16-tupled frequency millimeter-wave generation based on only two MZMs. Proc. SPIE 17th
International Conference on Optical Communications and Networks (ICOCN2018), 2019, 110482U.

28. Shimotsu S, Oikawa S, Saitou T, Mitsugi N, Kubodera K, Kawanishi T, Izutsu M. Single Side-Band Modulation Performance of a $\mathrm{LiNbO}_{3}$ Integrated Modulator Consisting of Four-Phase Modulator Waveguides. IEEE Photonics Technology Letters, 2001, 13(4):364-366.

29. Chow CW, Wang CH, Yeh CH, Chi S. Analysis of the carrier-suppressed single-sideband modulators used to mitigate Rayleigh backscattering in carrier-distributed PON. Optics Express, 2011, 19(11):10973-10978.

30. Loayssa A, Benito D, Garde MJ. Singlesideband suppressed-carrier modulation using a single-electrode electrooptic modulator. IEEE Photonics Technology Letters, 2001, 13(8):869-871.

31. Frankel MY, Esman RD. Optical singlesideband suppressed-carrier modulator for wide-band signal processing. IEEE Journal of Lightwave Technology, 1998, 16(5):859-863.

32. Cartaxo AVT. Small-signal analysis for nonlinear and dispersive optical fibers and its application to design of dispersion supported transmission systems with optical dispersion compensation. Proc. Inst. Elect. Eng., Optoelectronics, 1999, 146(5):213-222.

33. Corral JL, Marti J. General expressions for IM/DD dispersive analog optical links with external modulation or optical up-conversion in a Mach-Zehnder electrooptical modulator. IEEE Transactions on Microwave Theory and Techniques, 2001, 49(10):1968-1976.

34. Cheng L, Aditya S, Nirmalathas A. An exact analytical model for dispersive transmission in microwave fiber-optic links using MachZehnder external modulator. IEEE Photonics Technology Letters, 2005, 17(7):1525-1527.

35. Ferreira AA, Júnior, Coutinho OL, Martins CS, Fegadolli WS, Ribeiro JAJ, Almeida VR, Oliveira JEB. Effect of fiber optic chromatic dispersion on the performance of analog optical link with external modulation aiming 
at aerospace applications. Journal of Aerospace Technology and Management, 2013, 5(2):205-216.

36. Saleh BEA, Teich MC. Fundamentals of photonics. New York, John Wiley \& Sons Inc., 1991, 947 p.

37. James ET. Lithium Niobate Photonics. Boston, Artech House, 2015, 288 p.

38. Kong Eric SW (ed.). Nanomaterials, polymers, and devices: materials functionalization and device fabrication. New Jersey, John Wiley \& Sons Inc., 2015, 567 p.

39. Okayama H. Lithium Niobate Electro-Optic Switching. In: Optical Switching. Edited by ElBawab TS, Boston MA, Springer, 2006:39-81.

40. Wooten EL, Kissa KM, Yi-Yan A, Murphy EJ, Lafaw DA, Hallemeier PF, Maack D, Attanasio DV, Fritz DJ, McBrien GJ, Bossi DE. A review of lithium niobate modulators for fiber-optic communications systems. IEEE J. Select. Topics Quantum Electron., 2000, 6:69-82.

41. Belkin ME, Golovin VV, Tyschuk YuN, Kudzh SA, Sigov AS. Modeling optical intensity modulators based on MachZehnder interferometer by software tool of microwave circuits calculation NI AWR Design Environment. Russian Technological Journal, 2016, 4(5):3-16.

42. Min Xue, Shilong Pan, Yongjiu Zhao. Optical single-sideband modulation based on a dual-drive MZM and a $120^{\circ}$ hybrid coupler. Journal of Lightwave Technology, 2014, 32(19):3317-3323.

43. Agrawal GP. Nonlinear Fiber Optics. New York, Academic Press, 2013, 648 p.

44. Abramowitz M, Stegun IA. Handbook of Mathematical Functions With Formulas, Graphs, and Mathematical Tables, 10th ed: Wiley, 1972.

45. Andrews GE, Askey R, Roy R. Special Functions. Cambridge, U.K.: Cambridge Univ. Press, 2001.

46. Shcherbakov VV, Solodkov AF, Zadernovsky AA. Transmission of light intensity modulation signals in analog fiber-optic links. Radioelectronics. Nanosystems. Information
Technologies (RENSTT), 2016, 8(1):9-24; doi: 10.17725/rensit.2016.08.009.

47. Meslener GJ. Chromatic dispersion induced distortion of modulated monochromatic light employing direct detection. IEEE J. Quantum Electron., 1984, 20(10):1208-1216.

48. Schmuck H. Comparison of optical mmwave system concepts with regard to chromatic dispersion. Electron. Lett., 1995, 31:1848-1849. 